\title{
Universiteit
}

Leiden

The Netherlands

\section{Factors associated with mental health service need and utilization among unaccompanied refugee adolescents.}

Bean, T.; Eurelings-Bontekoe, E.H.M.; Mooijaart, A.; Spinhoven, P.

\section{Citation}

Bean, T., Eurelings-Bontekoe, E. H. M., Mooijaart, A., \& Spinhoven, P. (2006). Factors associated with mental health service need and utilization among unaccompanied refugee adolescents. Administration And Policy In Mental Health And Mental Health Services Research, 33, 342-355. doi:10.1007/s10488-006-0046-2

Version: $\quad$ Not Applicable (or Unknown)

License: $\quad$ Leiden University Non-exclusive license

Downloaded from: https://hdl.handle.net/1887/13183

Note: To cite this publication please use the final published version (if applicable). 


\title{
Factors Associated with Mental Health Service need and Utilization among Unaccompanied Refugee Adolescents
}

\author{
Tammy Bean · Elisabeth Eurelings-Bontekoe • \\ Ab Mooijaart · Philip Spinhoven
}

Published online: 28 April 2006

(C) Springer Science+Business Media, Inc. 2006

\begin{abstract}
This study is the first to address the need for mental health care (MHC) and the patterns of utilization of MHC services among Unaccompanied Refugee Minors (URM). Information concerning the well being, mental health need, and utilization of services of URM was collected from three informants, the minors themselves $(n=920)$, their legal guardians $(n=557)$, and their teachers $(n=496)$. The well-being, need and utilization of MHC services of URM was compared with those of a representative Dutch adolescent sample $(n=1059)$. The findings of this study indicated that URM that report a mental health care need (57.8\%) also report higher levels of emotional distress than Dutch adolescents who report a similar need for MHC (8.2\%). In addition, guardians and teachers detect emotional distress and mental health care needs in only a small percentage (30\%) of URM. The referral of URM to mental health care services does not appear to be driven by the reported needs of the URM, but by the need and emotional distress as observed and
\end{abstract}

T. Bean $(\square)$

Centrum '45, Rijnzichtweg 35, Oegstgeest, 2342 AX,

The Netherlands

e-mail: t.bean@centrum45.nl

E. Eurelings-Bontekoe $\cdot$ P. Spinhoven

Department of Clinical and Health Psychology, Leiden

University, Leiden, The Netherlands

\section{A. Mooijaart}

Department Psychology, Methods and Techniques, Leiden

Univeristy, Leiden, The Netherlands

P. Spinhoven

Department of Psychiatry, Leiden University, Leiden,

The Netherlands perceived by guardians. This resulted in the fact that $48.7 \%$ of the URM total sample reported that their need for mental health care was unmet.

Keywords Refugee $\cdot$ Adolescent $\cdot$ Mental health care $\cdot$ Utilization $\cdot$ Help-seeking

Refugees who have experienced many traumatic experiences can suffer from psychological distress for many years (Sack, Him, \& Dickason, 1999; Steel, Silove, Phan, \& Bauman, 2004). It has been documented that Mental Health Care (MHC) services have higher thresholds for adult refugees (Silove, Steel, McGorry \& Drobny, 1999) and for parental accompanied refugee adolescents and children than for the original population of the host country (Howard \& Hodges, 2000). Literature concerning the mental health needs of young refugees suggest that large scale therapeutic (social) care (Kohli \& Mather, 2003) or a low threshold to mental health services (Hodes, 2002) is needed to properly fulfill the needs of young refugees. Howard and Hodges (2000) found that the availability of interpreters and the liaison of psychiatric services with other social agencies facilitated help-seeking and utilization of MHC among young refugees. Unaccompanied Refugee Minors (URM) have been found to be at risk for the development of psychopathology (Felsman, Leong, Johnson, \& Felsman, 1990; Kinizie, Sack, Angell, Manson, \& Rath, 1986: Masser, 1992; Sourander, 1998) largely due to exposure to (sequential) traumatic experiences. While some have called this population "the most vulnerable of all" (Halvorsen, 2002), there is little known about their needs for mental health care and patterns of MHC utilization. This study will endeavor to address this very issue. As there is a dearth of studies on the MHC utilization of refugee children and adolescents, it is 
necessary to consult the general literature on theories concerning adolescents' MHC utilization to be able to examine the important issues surrounding the help-seeking process.

An epidemiological summary study reporting on the prevalence of psychopathology in adolescent populations, estimates that $15 \%$ of adolescents suffer from mental problems (Roberts, Attikison, \& Rosenblatt, 1998). Ezpeleta, Keeler, Erkanli, Costello, and Angold (2001) found a $12.9 \%$ prevalence rate in a representative sample of 1420 adolescents followed over 6 years. However, there remains a large gap between the adolescents who can be classified with a psychiatric disorder (or have sub-disorder symptoms levels-but are still functionally impaired) and the adolescents who actually utilize MHC services. The majority of the children and adolescents that suffer from mental health problems do not receive appropriate services (Leaf et al., 1996; US Department of Health and Human Services, 1999; Verhulst \& van der Ende, 1997). Several studies have shown that between 10 and $30 \%$ of the children and adolescents who need help actually utilize MHC services (Saunders, Resnick, Hoberman, Blum, 1994; Sournader et al., 2001). Zwaanswijk, Verhaak, Bensing, van der Ende, \& Verhulst (2003b) found in a representative Dutch adolescent population (11-18 years) that $10.5 \%$ of the adolescents reported having a mental health problem that was severe and $3.8 \%$ of the population self-reported an unmet MHC need (unmet need is defined as self-reporting a need for MHC services, but self-reporting not having obtained it). Flisher and colleagues (1997) found a higher percentage of unmet need, specifically $17 \%$, using more objective measures (presence of psychopathology and functional impairment). Other specific at-risk populations such as adolescents living in residential settings (Hukkanen, Sournader, Bergoth \& Piha, 1999), on child welfare (Burns et al., 2004), in foster care (Garwood \& Close, 2001; McMillen et al., 2005), inter-country adoptees (Tieman, van der Ende, \& Verhulst, 2005), or homeless adolescents (Bukner \& Baasuk, 1997; De Rosa et al., 1999) have been found to have higher levels of emotional and behavioral problems as well as greater psychological needs for MHC than normative adolescent populations.

From the known literature, it appears that there are distinct stages in the help-seeking process of adolescents (Cauce, Domenech-Rodriguez, \& Paradise, 2002; Srebrink, Cauce, \& Baydar, 1996); (a) Problem-recognition, (b) Decision to seek help (internal and external factors), and (c) Service selection/utilization. Problem recognition denotes the first step in seeking help; being aware that there is a problem. Srebink et al. (1996) stated that the severity level of psychological distress and behavioral problems are the most powerful predictor of problem recognition. This assumption has been confirmed in many studies (e.g. Saunders et al., 1994; Sourander et al., 2001; Zwaanswijk et al., 2003b).
As mentioned earlier, in several studies it has been documented that most of the children and adolescents that need professional mental health care (MHC) do not receive these services (e.g., Kataoka, Zhang, \& Wells, 2002). The help-seeking of children and adolescents occurs frequently in the social network in which they live and is usually not initiated by them (Srebink et al., 1996), but by their parents/caretakers. Many studies have found that parents play a crucial role (more important than the adolescent self) in adolescents MHC utilization (e.g., Kramer et al., 2004, Yeh \& Weisz, 2001). There is often a discrepancy between the need that adolescents report and the need that parents/ teachers report concerning MHC (Leaf et al., 1996). This discrepancy has been found to lead to adverse effects on the mental health of adolescents (Ferdinand, Van der Ende, $\&$ Verhulst, 2004). Family structure (1 or two parent) (e.g. Zwaanswijk et al., 2003a) and living situation (homeless, in home or out home placement) (Buckner \& Bassuk, 1997; McMillen et al., 2005; Sourander et al., 2004) have also been found to be important factors in MHC use. Since URM often live in residential settings in host countries where they do not receive $24 \mathrm{~h}$ supervision or only very limited supervision, the discrepancy between the need for MHC as felt by URM themselves and the need as perceived by significant adults could be even greater.

Furthermore, there are studies suggesting that externalizing problems are more prominent under adolescents and children that are referred or utilize MHC than internalizing problems (e.g., Wu et al., 1999, Yeh \& Weisz, 2001). Posttraumatic stress reactions and internalizing complaints such as depression and anxiety have been found to be prevalent forms of psychological distress among refugee children and adolescents (see Lustig et al., 2004 for a review) as result of extensive exposure to traumatic events. Experiencing stressful life events (abuse, loss) have been found to be strong predictors of MHC need and utilization in adolescent populations (Brugman, Reijnveld, Verhulst \& Verloove-Vanhorick, 2001; Burns et al., 2004; McMillen et al., 2005). In addition, several studies have found that ethnic minority groups underutilize MHC services as compared to adolescents from the dominant culture (Cauce et al., 2002; Flisher, Kramer, \& Grosser, 1997; Kataoka et al., 2002; Kodjo \& Auinger, 2004; Yeh, Hough, McCabe, Lau, \& Garland, 2004). These findings would seem to imply that the percentage of URM that would actually use MHC services might be lower than among the Dutch adolescent population due to under recognition of their psychological distress.

Individual subjective factors such as attitudes, beliefs (willingness), and knowledge (about mental health, available facilities) precede the actual help seeking and have been found to be vital aspects of the process of obtaining help (Sheffield, Fiorenza, \& Sofronoff, 2004; Verhulst \& 
Van der Ende, 1997; Zwaandijk et al., 2003a). Socialdemographic factors such as age and gender also appear to be important in the help-seeking process. Females have been repeatedly found to be more willing than boys to use MHC services (e.g., Garland et al. 2003; Sears, 2004). Older age of adolescent has been associated in several studies with more use of mental health services (e.g., Burns et al., 2004; McMillen et al., 2005; Sears, 2004) and with accurate problem recognition (Zwaanswijk et al., 2003b).

To address the issues outlined above that surround the help-seeking process of URM, this study will address the following questions (a) do the self-reported need for MHC, the willingness to use, the actual use of MHC services, and the self-reported unmet need of URM differ from that of a representative Dutch adolescent population and (b) which determinants facilitate the recognition of need and utilization of MHC services among URM?

\section{Method}

\section{Context of the Study}

In the years preceding 2001, there was a dramatic increase in the number of URM living in the Netherlands, peaking at 15,000 in 2001 . Many practical problems in referring unaccompanied minors to mental health care services were reported by the Nidos Foundation (legal guardian of all of the URM living in the Netherlands). Because of a lack of research on the mental health and mental health care utilization of URM, a national and longitudinal research project "Unaccompanied Refugee Minors and Dutch Mental Health Care Services" was started among URM living in The Netherlands and their guardians, teachers and professional mental health care providers in 2001. The goal of the project was to determine the severity of psychological distress of URM, their need for mental health care, and the availability of mental health care services for this population. Ethical approval was given by the Medical Ethics Committee of the Leiden University Medical Center, Leiden University to conduct the study.

\section{Samples}

URM Sample $(n=920)$

Approximately 4,000 URM were randomly selected from the Central Register of Nidos. Information about the study and permission waivers (available in translated versions) were sent to the guardians to discuss with the URM. Both the minor and his/her guardian gave written permission for the URM to participate. Roughly 2,300 URM permission waivers were returned; 1300 (57\%) wished to participate,
$15 \%$ refused, $12 \%$ did not participate for a wide range of practical reasons, $9 \%$ were transferred, and $7 \%$ turned out to be untraceable. However, there were no significant differences found between the URM that did participate and the URM that did not in gender (ns), age (ns), and country of origin $(n s)$. A total of 920 URM participated in the study. The final sample was representative in all of the main characteristics of the total URM population aged 1218 year old in 2002 in the Netherlands. The URM came from 48 countries. Two-thirds of the sample had been living in the Netherlands for a period of 18 months or less. In addition to the questionnaires mentioned above, an interview regarding mental health care was individually administered. Three research assistants administered the questionnaires during $1 \mathrm{~h}$.

\section{Dutch Normative Sample $(n=1,059)$}

Pupils who were peers of the URM from 10 secondary and three trade schools throughout the Netherlands (schools had also taken part in the URM study) participated and functioned as a control group for the URM sample. Two weeks prior to administration of the instruments, informed consent letters were sent to the parents and adolescents asking for voluntary and anonymous participation (27 students abstained from participation). The assessment of the Dutch sample took approximately 15 minutes per class.

\section{Procedures}

There is one foundation that has the legal guardianship of all of the unaccompanied minors that reside in the Netherlands, The Nidos Foundation. This foundation has offices throughout the entire country and has almost 20 years of experience in working with unaccompanied minors. Two information packages (one for guardian and one for teacher) were sent to the supervisors of each regional office for each guardian that was responsible for one of the 920 unaccompanied minors that took part in the study. The guardians received a letter with the questionnaires informing them about the study and giving instructions concerning how the questionnaires should be filled in. The guardians were instructed in the letter and by their supervisors that they could fill in the questionnaire or ask a staff member of the living unit/ foster parent of the unaccompanied minors to do so. However, the guardian remained responsible for retuning the completed questionnaires to their supervisors which in turn sent back all the completed questionnaires from the regional office. For the first assessment period, 557 questionnaires were returned from the guardians.

The guardian was also responsible to send the information package to the teacher. Enclosed in the information 
package for the teacher, was a letter describing the project, questionnaires and a stamped and addressed enveloped in order to enable the teacher to return the completed questionnaires directly. The teachers received a letter with the questionnaires informing them about the study and giving instructions concerning how the questionnaires should be filled in. For the first assessment period, 496 questionnaires were returned from the guardians.

\section{Instrument Descriptions}

The self-report questionnaires were translated into the most prevalent languages of URM in the Netherlands: Albanian, Amharic, Arabic, Badini, Chinese, Dari, Dutch, English, Farsi, French, German, Mongolian, Portuguese, Russian, Servo-Croatian, Soerani, Somali, Spanish, and Turkish. The literal terms of the Likert scale (not $=1$, little $=2$, much $=3$, very much $=4$ ) was improved by using colored circles of increasing size. Items were simplified to adapt the questionnaires to the language abilities of this population, and the questionnaires were translated and presented in a bilingual form.

The cross-cultural validation process for the three questionnaires followed the five dimensions of equivalence for cross-cultural validation of an instrument proposed by Flahtery et al. (1988). The five dimensions are (a) content equivalence which determines whether each item is equally relevant for the culture(s), (b) semantic equivalence is an item-by-item analysis attempting to convey the original meaning of each item in the adapted version(s), (c) technical equivalence refers to whether the data collection method (e.g., self-report survey, in-person interview) yield comparable results in each culture; (d) criterion equivalence is when the interpretation of the measurement remains the same when norms are compared in each culture, and (e) conceptual equivalence refers to whether the same theoretical construct is being measured in each culture.

All written forward translations were done by professionally employed translators. Every translation was controlled for grammatical and idiomatic errors on two different occasions by two different translators. The translated questionnaires were reviewed orally with professional interpreters who where regularly involved in treatment sessions of traumatized adult refugees to control for the quality of the translations, to ensure that the original meaning was conveyed in the items, and to attempt to achieve semantic equivalence.

The Hopkins Symptom Checklist-37 for Adolescents (HSCL-37A) (Bean, Derluyn, Eurelings-Bontekoe, Spinhoven, 2004a) measures internalizing distress (anxiety and depression symptoms) and externalizing behavior (traumarelated "acting-out"). The psychometric properties have been investigated among a culturally diverse adolescent population and appeared to be satisfactory to good (Bean et al., 2004a). Internal reliability for the URM sample for the total scale, internalizing and externalizing behavior subscales was respectively .91, 92, and .69. Twelve-month test-retest reliability for the total scale was analysed with a Pearson correlation coefficient and appeared to be satisfactory $(r=.63, \mathrm{P}<.001)$ (Bean et al., 2004a). Intermeasure correlations with the total scores of the RATS and SLE were respectively $.77(\mathrm{P}<.001)$ and $.38(\mathrm{P}<.001)$ (Bean et al., in press). Using a confirmatory factor analysis, the two-factor structure, internalizing and externalizing, was verified in the URM sample with a loss of only .4\% of the explained variance. URM who reported a need for MHC, reported using MHC, or were referred to MHC by their guardian reported a score of 52 or higher on the internalizing scale which corresponds with the 90th percentile for Dutch adolescents.(Bean et al., 2004a). URM who reported a need for MHC, reported using MHC, or were referred to $\mathrm{MHC}$ by their guardian reported a score of 19 or higher on the externalizing scale which corresponds with the 90th percentile for immigrant/and refugee adolescents and the 65th percentile for Dutch adolescents (Bean et al., 2004a).

The Stressful Life Events (SLE) checklist (Bean, Derluyn, Eurelings-Bontekoe, Broekaert, \& Spinhoven, in press) was used to assess the number and type of stressful event(s) that was experienced. The SLE consists of 12 dichotomous (yes/no) questions and an open question on the occurrence of stressful life events of relevance for adolescent refugee minors (e.g. "Have you ever experienced a war or an armed military conflict going on around you in your country of birth?" or "'Has someone ever hit, kicked, shot at or some other way tried to physically hurt you?'). Having experiencing a traumatic event is the first criterion of cluster A1 of the DSM-IV for PTSD (American Psychiatric Association, 1994). The overall average total score of 6.5 of the SLE for URM has been replicated in 5 independent studies (Bean, et al., 2004b).

The Reactions of Adolescents to Traumatic Stress (RATS) (Bean, et al., in press) is a self-report questionnaire developed to assess posttraumatic stress reactions defined in the DSM-IV (American Psychiatry Association, 1994) with culturally diverse adolescents. The RATS consists of 22 items that correspond directly to the B (intrusion), C (numbing/avoidance), and D (hyper-arousal) criteria of the DSM-IV for PTSD. Items were adapted to measure symptoms of intrusion, numbing/avoidance and hyper-arousal in adolescents, especially adolescent refugees. The psychometric properties have been investigated among culturally diverse adolescent populations and per language version of the RATS and appear to be satisfactory to good (Bean et al., 2004c). Internal reliability for the URM sample for the total scale, and intrusion, numbing/ 
avoidance and hyper-arousal subscales was respectively $.88, .85, .69$, and .73 . Twelve-month test-retest reliability for the total scale was analysed with a Pearson correlation coefficient and appeared to be satisfactory $(r=.61$, $\mathrm{P}<$.001) (Bean, et al., in press). Using a confirmatory factor analysis, the three-factor structure was verified in the URM sample with a loss of only $3 \%$ of the explained variance (Bean et al., 2004c). URM who reported a need for MHC, reported using MHC, or were referred to MHC by their guardian reported a score of 52 or higher on the RATS total scale which corresponds with the 97th percentile for Dutch adolescents (Bean et al., 2004c).

\section{Mental Health Questionnaire for Adolescents}

The self-perceived need for, knowledge of and satisfaction with MHC services was measured using an interview of 23 items. The interview was individuality conducted with the URM after they had filled in the other three questionnaires to ensure that the questionnaire would be filled in properly. The research assistants always stressed that the questions were about receiving help for problems regarding "thinking and feeling" and not about practical problems. This interview was translated in the above mentioned languages so that the URM could read along in their own language if that was necessary or for clarification. Examples of the questions are: Do you think that you have problems (emotional) that you need help for?, Would you like to contact someone that could help you (with your emotional problems)?, Have you already been to a '(mental) health professional' (for your emotional problems)?. Dutch adolescents were asked to only fill-in these same three questions so that comparison could be made between the two groups.

\section{Child Behavioral Checklist (CBCL)}

The Dutch version of the CBCL/4/18; 1991 Profile(Achenbach, 1991)-Dutch translation (Verhulst, van der Ende \& Koot, 1996) was used to standardize the assessment of the behavior and emotional problems of unaccompanied minors through the observations of guardians. The CBCL has been found to be a reliable and valid instrument to be utilized by other informants than parents (Albrecht et al., 2001; Dutra et al., 2004). The CBCL scores for this study were dichotomized. The cutoff point was a $T$ score of 60 or above for both the internalizing and externalizing scales. This cut off point has been established among Dutch adolescents and indicates a score which falls on or above the clinical borderline range (Verhulst et al., 1996). The validity and reliability of the Dutch CBCL for normative and clinical populations is thoroughly described in Verhulst et al. (1996). The psychometric properties for the CBCL in this study did not differ from those of Verhulst and colleagues (Bean, Mooijaart, Eurelings-Bontekoe \& Spinhoven, in press).

\section{Mental Health Questionnaire for Guardians}

The need for mental health care among the unaccompanied minor as perceived by the guardian, the referral process to mental health services and the satisfaction with the utilized mental health care was measured using a checklist of 23 items. The guardians were also asked to fill in 10 questions about themselves and their experience with working with adolescents.

\section{Teacher's Report Form (TRF)}

The Dutch version of the TRF 4/18; 1991 Profile(Achenbach, 1991)-Dutch translation (Verhulst, van der Ende \& Koot, 1997) was used to standardize the assessment of the behavioral and emotional problems of unaccompanied minors through the observations of teachers. The TRF scores for this study were dichotomized. The cutoff point was a $T$ score of 60 or higher for both the internalizing and externalizing scales. This cut off point has been established among Dutch adolescents and indicates a score which falls on or above the clinical borderline range (Verhulst et al., 1997). The validity and reliability of the Dutch CBCL for normative and clinical populations is thoroughly described in Verhulst et al. (1997). The psychometric properties for the TRF in this study did not differ from those of Verhulst and colleagues (Bean, Mooijaart, Eurelings-Bontekoe \& Spinhoven, submitted).

\section{Mental Health Questionnaire for Teachers}

The need for mental health care among the unaccompanied minor as perceived by the teacher and the referral process to mental health care services were measured using a checklist of 6 items. The teachers were also asked to fill in 10 questions about themselves and their experience with working with adolescents.

\section{Statistical Analysis}

Descriptive statistics were used to give summary descriptions of the socio-demographic characteristics of the samples (Table 1). Differences in socio-demographic and MHC variables between URM and Dutch samples were determined by using the Chi-square test and $t$-test. Effect sizes were calculated using Cohen's $d$ (Cohen, 1988). A maximum of ten percent of the missing items was allowed 
Table 1 Summary characteristics

\begin{tabular}{|c|c|c|c|c|}
\hline & Unaccompanied minor & Dutch adolescents & Guardians & Teachers \\
\hline$N$ & 920 & 1059 & 557 & 496 \\
\hline \multicolumn{5}{|l|}{ Gender } \\
\hline Male & $71.3 \%$ & $57.0 \%$ & $22.4 \%$ & $31.9 \%$ \\
\hline Female & $28.7 \%$ & $43.0 \%$ & $77.6 \%$ & $68.1 \%$ \\
\hline \multicolumn{5}{|l|}{ Age in years } \\
\hline Mean & 15.48 & 15.72 & 36.27 & 45.97 \\
\hline SD & 1.52 & 1.54 & 8.96 & 8.72 \\
\hline Range & $10-18$ & $10-21$ & $20-64$ & $23-64$ \\
\hline \multicolumn{5}{|l|}{ Country of Origin } \\
\hline Netherlands & $0.0 \%$ & $89.8 \%$ & $78.9 \%$ & $90.3 \%$ \\
\hline Angola & $43.9 \%$ & & & \\
\hline Iran/Afghanistan/Iraq & $4.4 \%$ & & & \\
\hline Eritrea/Ethiopia & $2.7 \%$ & & & \\
\hline Somalia & $2.1 \%$ & - & & \\
\hline Sierra Leone & $7.9 \%$ & & & \\
\hline Guinea & $6.7 \%$ & & & \\
\hline Other African countries & $14.0 \%$ & & & \\
\hline China/Tibet & $8.6 \%$ & & & \\
\hline Other countries & $9.6 \%$ & $10.2 \%$ & $21.1 \%$ & $7.8 \%$ \\
\hline Referral of URM to MHC services & & & $11.7 \%$ & \\
\hline Need for MHC & $57.8 \%$ & $8.2 \%$ & (URM) $18.1 \%$ & (URM) $29.8 \%$ \\
\hline Willingness for MHC & $72.2 \%$ & $11.7 \%$ & & \\
\hline Utilization of MHC & $12.7 \%$ & $16.1 \%$ & & \\
\hline Unmet need & $48.7 \%$ & $4.5 \%$ & & - \\
\hline
\end{tabular}

to still be able to extrapolate the total or subscale scores. Probability values for all analyses were set at $<.05$. The statistical power analysis was performed in relation to the variable in which the smallest number of subjects was expected, i.e. self-perceived unmet need. With respect to the other variables (reported need for MHC services, willingness for MHC services, and reported use of $\mathrm{MHC}$ services), larger number of subjects are to be expected (Sears, 2004; Sheffield, Fiorenza, Sofronoff, 2004; Zwaanswijk et al., 2003b). On the basis of previous research (Zwaanswijk et al., 2003b) it was hypothesized that at least approximately $5 \%$ of the Dutch adolescents would report an unmet need and that the proportion of URM reporting an unmet need would be at least twice as high. In order to detect a between group difference with a medium effect size (Cohen's $d=.50$ ) with power of $80 \%$ and a twotailed significance level of $<.05$, a minimum of 50 Dutch adolescents and 100 URM with an unmet need are required. Subsequently, at least 1000 normal Dutch adolescents and 1000 URM would need to be included in the study in order to obtain the minimum number of subjects which report an unmet need.

Multivariate logistic regression was used to assess the correlates of the binary dependent variables in the following models; (1) Self-reported need for services, (2) Guardian's report; need for services, (3) Teacher's report; need for services, (4) Service use; URM report, (5) Referral; Guardian report, and (6) Unmet need of URM.
The binary independent variables that appeared in all models are: gender $(1=$ female $)$, age $(1=16$ years or older), family member living in the Netherlands $(1=$ has family member), length of stay in the Netherlands ( $1=13$ months or longer), receives $24 \mathrm{~h}$ adult supervision ( $1=$ receives less than $24 \mathrm{~h}$ supervision), number of years of school attendance ( $1=6$ years or more), total number of reported Stressful life events (SLE's) $(1=7$ or more SLE'S), score falling above preliminary cut-off for total RATS ( $1=52$ or higher), score falling above preliminary cut-off for HSCL-37A internalizing ( 1 = 52 or higher), and score falling above preliminary cut-off for externalizing score $(1=19$ or higher). In addition, the CBCL internalizing and externalizing reports (T-scores $>60)$ from the guardian and the TRF internalizing and externalizing reports $(T$-scores $>60)$ from the teachers, were entered into the regression analysis for respectively the (2) guardians report of need and (3) teacher report of need because these variables were relevant in informant specific models.

\section{Results}

Demographic Information about Unaccompanied Minors and Teachers

The distribution of gender across the two adolescent samples will be addressed first (Table 1). The distribution 
of boys and girls in the URM sample deviated from that in the Dutch sample $\left(x^{2}(1)=53.04, P<.001\right)$ with a higher proportion of boys in the URM sample. The results from a $t$-test showed that the mean age for both groups did not differ significantly from each other $(t(1942)=1.21$, ns).

Table 1 represents the demographic background information for the URM, Dutch sample, URM teachers and URM Guardians. The most frequent countries of origin were Angola, Sierre Leone, Guinea and China. Most of the unaccompanied minors came from Africa (80.8\%). $44.9 \%$ of the unaccompanied minors sample had received educational training for more than 5 years.

On the average one guardian filled in a questionnaire for 2 unaccompanied minors, however because of the large and quick turnover rate of guardians and rapid transfers of the URM, it was not possible to establish the exact number of guardians that took part in the study. The guardian population consisted of mostly females $(77.6 \%)$ with a mean age of 36 years. The greater majority of guardians were born in the Netherlands. A large portion of the guardians had received the equivalent of a Bachelor's degree in Social Work.

The teachers could reply anonymously if they chose to do so. Therefore, it was also not possible to establish the exact number of teachers that took part in the study. We were able to estimate that approximately 400 teachers took part in the study. The teacher population consisted of mostly females $(68.1 \%)$ with a mean age of 46 years. The greater majority of teachers were born in the Netherlands. Most of the teachers had received the equivalent of a Bachelor's degree in Teaching.

\section{Self-reported Mental Health need of URM}

About $57.8 \%$ of the URM reported a need for help for their emotional distress (Table 1). This percentage is in sharp contrast with the Dutch adolescents of which only $8.2 \%$ reported such a need. Twenty-one percent of the guardians of URM and almost $30 \%$ of the teachers of URM reported a need for professional MHC for the URM in their care. There was $11.7 \%$ of the URM referred to MHC services by the guardian. Moreover, $12.7 \%$ of the URM had used a type of professional MHC in the Netherlands (16.1\% utilization rate for Dutch adolescents). Using the same definition for "unmet need" as Zwaanswijk and colleagues (2003b) (self-reporting a need for MHC services, but not having obtained it) $4.5 \%$ of the total Dutch adolescent sample reported an unmet need. This proportion does not deviate extensively from the $3.8 \%$ found by Zwaanswijk and colleagues (2003b). In great contrast, $48.7 \%$ of the total URM population reported having an unmet MHC need.
In examining only the cases in which information concerning the need for MHC for URM was supplied by all three informants ( $n=288$ ), the guardian and/or teacher only perceived the need for MHC among $30.6 \%$ of the URM that themselves had reported a need for MHC. In addition, the guardian and/or teacher only perceived the need for MHC among $12.2 \%$ of the URM that themselves had reported being uncertain if they needed help. In only $6.3 \%$ of the cases there was agreement between all three informants (URM, teacher and guardian) about the need for MHC.

Comparisons in Need, Unmet Need, Willingness, and Actual Use of MHC

In Table 2, the between group differences (Dutch normative adolescents and URM) are shown for the HSCL-37A total, internalizing, externalizing scales, the total number of experienced SLE's and the RATS questionnaire. URM clearly reported significantly higher mean scores for the HSCL-37A total, internalizing, and RATS (large to very large effect sizes; range .66-1.67) than Dutch normative adolescents. URM further reported experiencing two times as many SLE's as Dutch adolescents. However, Dutch normative adolescents reported higher externalizing scores than URM $(d=.68)$.

Within the URM sample as well as in the Dutch adolescent sample, the severity of complaints and total number of reported SLE's was significantly higher $(P<.001)$ among those adolescents who reported need, utilization of some form of mental health are, or had an unmet need than among adolescents that had no self-reported need, did not use services and had no unmet need (data not shown). The only exception was the HSCL-37A externalizing scale.

Table 2 Comparisons between total URM and Dutch adolescents' samples

\begin{tabular}{|c|c|c|c|c|}
\hline Scale & $N$ & $M(\mathrm{SD})$ & $t(\mathrm{df})$ & $d$ \\
\hline \multicolumn{5}{|c|}{ HSCL-37A Total } \\
\hline URM & 835 & $66.33(14.70)$ & \multirow[t]{2}{*}{$13.74 * * *(1477)$} & .66 \\
\hline Dutch & 1058 & $58.00(10.73)$ & & \\
\hline \multicolumn{5}{|c|}{$H S C L-37 A$ internalizing } \\
\hline URM & 828 & $50.78(12.93)$ & \multirow[t]{2}{*}{$4.04 * * *(1884)$} & \multirow[t]{2}{*}{1.0} \\
\hline Dutch & 1058 & $39.81(9.26)$ & & \\
\hline \multicolumn{5}{|c|}{ HSCL-37A externalizing } \\
\hline URM & 852 & $15.49(3.27)$ & \multirow[t]{2}{*}{$-15.25 * * *(1892)$} & .68 \\
\hline Dutch & 1058 & $18.19(4.46)$ & & \\
\hline \multicolumn{5}{|c|}{ Stressful life events total } \\
\hline URM & 894 & $6.17(2.61)$ & \multirow[t]{2}{*}{$34.46^{* * *}(1476)$} & \multirow[t]{2}{*}{1.3} \\
\hline Dutch & 1058 & $3.05(2.18)$ & & \\
\hline \multicolumn{5}{|c|}{ RATS total } \\
\hline URM & 799 & $49.21(11.54)$ & \multirow[t]{2}{*}{$28.31 * * *(1745)$} & \multirow[t]{2}{*}{$1.6^{7}$} \\
\hline Dutch & 1059 & $32.17(9.09)$ & & \\
\hline
\end{tabular}

*** $P<.001$ 
The univariate tests revealed that URM that reported need, willingness to receiving MHC, utilization of some form of mental health care, or URM with an unmet need had significant higher mean scores (medium to very large effect sizes; range .41-1.25) than the Dutch adolescents on the HSCL-37A internalizing, Total number of Stressful life events, and RATS total scales (see Table 3). Regarding the externalizing scale of the HSCL-37A, Dutch adolescents who reported a MHC need reported significantly higher mean scores (large effect sizes; range .76-1.00) across all the variables (need, willingness, MHC use and unmet need) than URM. There were no significant differences found between the URM and Dutch adolescents HSCL-37A total scores on need, use or unmet need variables. Although, there was a significant difference in HSCL-37A total means scores of URM and Dutch adolescents for willingness, the effect was of medium size (.44).

The lack of a significant difference between the two samples on the HSCL-37A total scores (Dutch and URM adolescents who reported needing and were willing to receive MHC) is apparently due to the different profiles of emotional and behavioral problems that URM and Dutch adolescents seek help for, which cancel each other out in the total score of the HSCL-37A.

\section{Correlates of Mental Health care Service Use}

First, the binary multivariate logistic regression analyses were calculated (Model 1-See Table 4) using the determinants gender, age, family member living in the Netherlands, length of stay in the Netherlands, receives $24 \mathrm{~h}$ adult supervision (not shown in Table 4), number of years of school attendance (not shown in Table 4). In addition, the internalizing and externalizing scores from the informant specific questionnaires (HSCL-37A-URM; CBCL-guardian; and TRF-teacher) were only entered into the specific informant regression model.

There were six individual dependent variables analyzed using logistic regression; (1) Need; self perceived, (2) Need; Guardian's report, (3) Need; Teacher's report, (4) Referral; Guardian, (5) Service use; and (6 Unmet need. Overall, the independent variables in each regression model predicted significantly 5 of the dependent variables well. Only for the dependent variable (5) Service use; URM report, the regression model was not found to be significant, which implies that the factors in the model are not sufficient in predicting the service use of URM. Only URM scores falling above the preliminary cutoff for the HSCL-37A internalizing scale were a key predictor in this model (Table 4).

For both dependent variables, 'Self-reported need' and 'Unmet need' of URM, the same independent variables were found to have significant odds ratios in both models






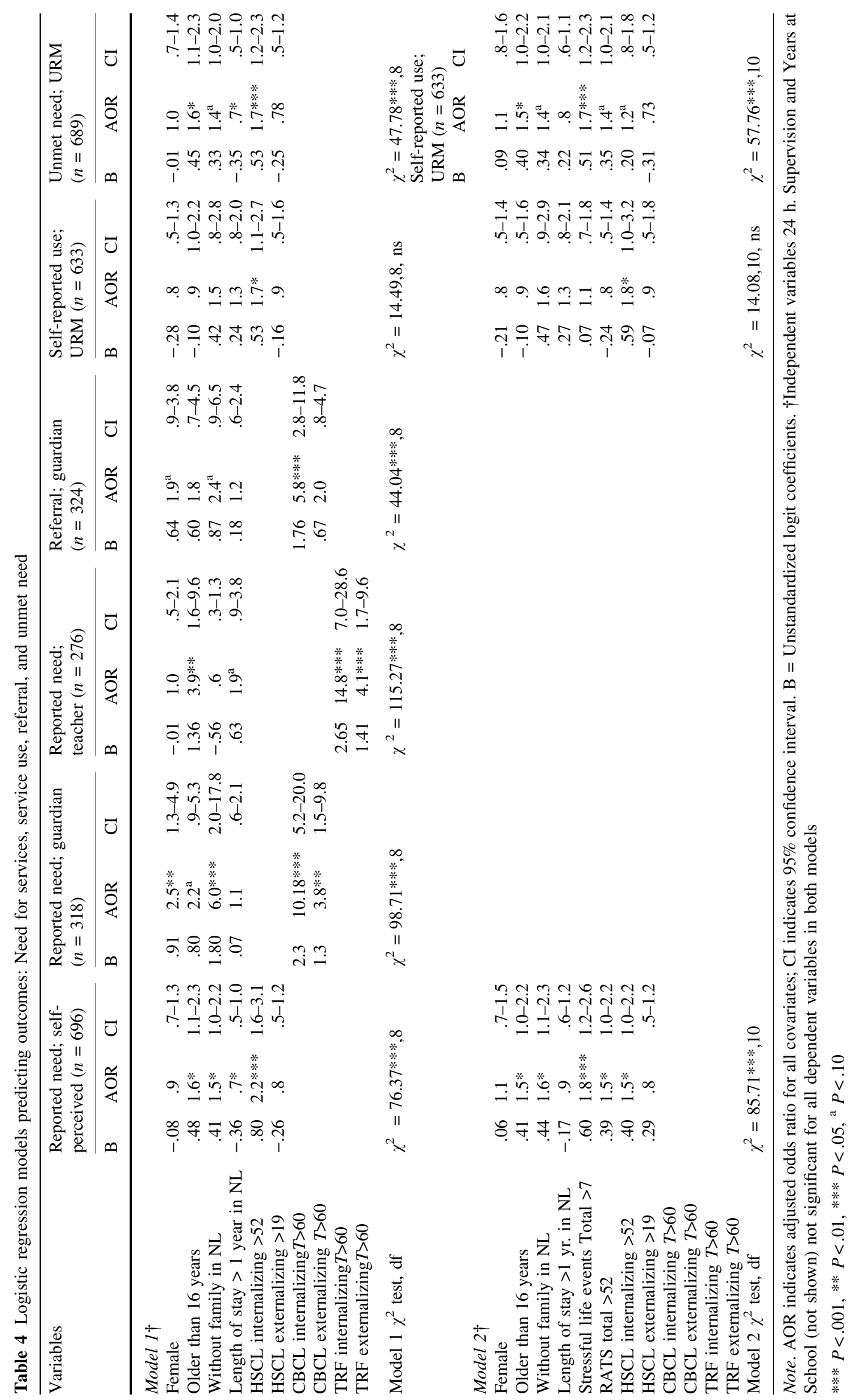


(i.e. older age, having family living in the Netherlands, scores falling above the preliminary cutoff for the internalizing subscale of the HSCL). In other words, if URM were older, had no family member living in the Netherlands, had scores falling above the preliminary cutoff for the internalizing subscale of the HSCL they were more likely to have reported a need or have had an unmet need than URM that were younger, had a family member living in the Netherlands and did not report high internalizing scores.

When examining the findings of the guardian's reports of need for professional MHC services, the significant independent variables in the model were being female, older age, living in the Netherlands without a family member, CBCL internalizing and externalizing scores. The CBCL internalizing scores falling above/on the borderline range and living in the Netherlands without a family member were the most robust predictors in the model meaning that if a guardian perceived the emotional distress of the URM to be great and the URM had no family in the Netherlands that they were more likely to be found by the guardian to have a need for professional MHC.

The internalizing and externalizing TRF scores falling above in/or above the borderline range had the highest odds ratio's in the model and therefore the most significant predictors of the teacher's report of need for services. Furthermore, as can be seen in Table 4 older age and length of stay in the Netherlands were also significant variables in predicting whether the teacher reported that a URM was in need of professional MHC.

Finally, in the regression model in which the referral to MHC services by the guardian was examined, being female, having no family living in the Netherlands, and the internalizing CBCL scores falling above the established borderline range were of predictive importance. Moreover, the variable CBCL internalizing scores had the highest odds ratio implying that when the guardian considered the emotional distress of the URM to be elevated, they referred URM to professional mental health care services.

\section{Entering Self-reported Traumatic Stress Experiences} and Reactions of URM into the Regression Models

Several independent variables were added to the regression models (Regression model 2 in Table 4) to predict MHC need and service use. In these analyses, the RATS scores and SLE total scores were added to the URM specific need and utilization variables to see if the odds ratio would be altered.

In respect to the dependent variable 'Self-reported service use', the only significant predictor remained the HSCL-37A internalizing scores after adjusting for the
RATS total score and number of SLE and the model again was not found to be significant.

Adjusting the dependent variables URM 'Self-perceived need' and 'Unmet need', by entering of the new independent variables (RATS and SLE) into the model had a significant effect on the model. Reporting to have experienced 7 or more SLE's was found to be the most robust predictor in both models ('Self-perceived need'; AOR $=1.8(95 \%$ CI: $1.2-2.6)$ and 'Unmet need'; AOR $=1.7(95 \%$ CI: $1.2-$ 2.3). In addition, age, without family, RATS total scores and HSCL-37A internalizing scores were found to be significant independent variables for both models. The odds ratio for age and family did not change after the adjustment for RATS and SLE total scores. However, the odds ratios for HSCL-37A internalizing scores were reduced for 'Selfperceived need' and 'Unmet need' respectively $22 \%$ $\left\{[(2.2 / 1.8)-1]^{*} 100\right\}$ and $42 \%\left\{[(1.7 / 1.2)-1]^{*} 100\right\}$.

\section{Discussion}

This study is the first to document the need for mental health care, the unmet need and the correlates of service utilization of unaccompanied refugee minors. Unique to this study is that a large URM sample was compared to Dutch adolescent peers and that information concerning the MHC need and service utilization of URM was provided by several informants, i.e., legal guardians and teachers. Approximately $60 \%$ of the URM had a need for MHC with only a small percentage $(11.7 \%)$ ever receiving professional mental health care services. This perceived need is very large when compared with rates among Dutch peers, but very comparable to other at-risk adolescent populations (e.g., Burns et al., 2004; Garland et al., 2003).

The results of this study further show that the severity of the psychological distress of URM that have a need, unmet need or that have utilized services bypasses that of Dutch peers. In the regression analyses, it was quite clear that the severity levels reported by URM and guardians play a very important role in perceived need and referral to MHC services confirming the assumption of Sebrink, and colleagues (1996). However, for the URM the number of experienced stressful life events was the most crucial predictor, as well as their symptom levels, in their self-reported need (or unmet need) for professional help. This result has also been found in the studies among children in welfare services of McMillen et al. (2005) and Burns et al. (2004).

Unfortunately the regression models of guardians and teachers could not be adjusted for the number of experienced stressful life events and psychological problems (traumatic stress reactions) reported by the URM 
themselves since the guardians and teachers were unaware of this information. In future studies, inclusion of this information from guardian/caregivers and teachers concerning the severity of traumatic stress reactions of URM and the recorded (verified) stressful life events that they have experienced could give more insight if guardians and teachers take the these factors into consideration when deciding if a URM needs professional MHC. For both teachers and guardians, their own appraisals of the severity of emotional distress of the URM were the most robust predictors of the need of professional MHC for URM. Furthermore, the internalizing scores from the informant specific questionnaires were better predictors of URM utilization of MHC than externalizing scores.

Younger age and having a family member living in the Netherlands were found in this study to be protective factors in keeping URM from having a need or using MHC services. The effect of age has been previously found in other studies to be confounded by other factors such as type of living situation (e.g., McMillen et al., 2005) and number of experienced stressful life events (e.g., Smith, Perrin, Yule, Hacam, \& Stuvland, 2002). For this special population, governmental policies (financial government assistance stops at age 18) could also be a confounding factor. The association between confounding factors and age is not clear in this study and will need to be further examined in future investigations. Furthermore, the shielding effect of living with family members is also not new (Sack, Angell, Kinzie, \& Rath, 1986). A (perceived) supporting social network has been found to be negatively associated with the use of professional mental health care services (Ten Have, Vollenbergh, Bijl, \& Ormel, 2002). Living longer than one year in the Netherlands was also found to be an important factor in perceived need of URM and teachers. This result can indicate that when URM are living for longer periods in the Netherlands, they are more capable of perceiving their need and conveying it to others, perhaps due to acquiring skills that enable them to know when to ask for help. Ciarrochi and colleagues (2002) have proposed that teaching adolescents how to effectively identify and manage emotions will lead to positive help-seeking behavior such as a better ability to estimate when help is needed from their social network.

\section{Methodological Challenges}

This study was limited and methodical challenging because of the unstable situation in which the URM live (frequent transfers, limited supervision) and the extreme diversity of the population. The psychological measurements that have been used in this study have been thoroughly examined in the manuals and articles mentioned earlier reporting on their validity and reliability with diverse adolescent populations. It was not apparent in the data that there should be doubt that adolescents from one culture perceived the questions differently than another; however individual differences that did not appear in the data could have taken place.

A high non-response rate can be expected in this kind of study. Although the non-response group was quite large, at least on an objective demographic level, there were no apparent differences in the study sample and non-response group. However, here again individual subjective differences that did not appear in the data could have been present but were not apparent. For example, URM that did not want to take part in the study could have been exhibiting avoidance or withdrawn behavior to eliminate thinking or being confronted with their distress and/or past adverse experiences. Furthermore URM with more open and agreeable personality traits might have been more willing to take part in this study in contrast to more resistant or unwilling URM. Although 920 instead of at least 1000 URM were included, the statistical power of the present study to detect a difference with a medium effect size between groups with the smallest size (as expected based on participants with self-perceived unmet need) was still very high $(89 \%)$ due to the larger than expected percentage of URM with self-perceived unmet need of MHS services.

No psychiatric interview was administered to verify the high severity levels found with the questionnaires due to the absence of translated standardized diagnostic interview in the languages of the URM in this study and the complex semantic challenges of ensuring "the semantic and psycholinguistic equivalence of psychiatric symptoms across cultures" (Cheng, 2001). No established mental health questionnaires for a culturally heterogeneous adolescent population, teachers or guardians in the Netherlands were known to the authors, which necessitated the creation of new measures. Furthermore, the data do not reveal that there was a misunderstanding of the nature and content of the perceived mental health care need among URM. However, in individual cases, misinterpretation of the question might have influenced the results concerning the self-reported need among URM of MHC.

It is recommended, as in other studies that have evaluated the mental health care needs of at-risk populations (Burns et al., 2004; Garwood \& Close, 2001; McMillen et al., 2005), that periodical mental health assessments of URM be put into practice to ensure that their MHC needs are recognized. The ramifications of this study also indicate that the self-reported need and severity level of emotional distress of URM should be taken seriously in the decision-making process for treatment or intervention. Disagreement between informants should not be ignored, but addressed as it can be crucial information to treatment 
(i.e., maladaptive avoidance behavior; relationship discord) (Kramer et al., 2004; Ferdinand et al., 2004).

Furthermore, the findings of this study draw attention to the need to provide adequate living situations with adult supervision for these adolescents to attempt to alleviate daily stressors that can exacerbate their fragile mental health. URM no longer have parents that can help them learn to regulate their intense emotional reactions to the situations that they have experience or in other words to become emotional competent. The current significant adults in the lives of these young people need to be aware of their great need for MHC and high levels of emotional distress. As professionals, it is important that the caregivers of URM are adequately trained to give these adolescent psychological "tools" for their toolbox (active coping skills) (Weisz \& Hawley, 2002). Receiving low threshold psycho-education (residential or school-based) programs on active coping skills can empower URM to manage the trauma and stress they have had to endure and reduce the great (unmet) need these young people have reported for mental health care. The school setting has been found to be experienced among refugee adolescents as a secure place (e.g, Sack et al., 1986). School-based interventions continue to play an important part in making mental health care services available at one point to a broad population (Farmer, Burns, Phillips, Angold, \& Costello, 2003) and have worked well for refugee adolescents (Rousseau, Drapeau, Lacroix, Bagilishya, \& Heusch, 2005). In conclusion, it is paramount to the emotional development of URM that governmental agencies and mental health care services work together to implement policies that acknowledge the psychological suffering and the great need for professional MHC of this at-risk population.

Acknowledgements The Dutch studies were supported by Achmea Victim and Society Foundation and the Health Research Development Counsel (ZON-Mw)

\section{References}

Albrecht, G.,Veerman, J. W., Damen, H., \& Kroes, G. (2001). The Child Behavior Checklist for group care workers: A study regarding the factor structure. Journal of Abnormal Child Psychology, 29, 83-89.

American Psychiatry Association (1994) Diagnostic and statistical manual of mental disorders (4th ed). Washington, D.C.: American Psychiatry Association.

Bean, T., Derluyn, I., Eurelings-Bontekoe, E. H. M., Broekaert, E., \& Spinhoven, P. (in press). Validation of the multiple language versions of the reactions of adolescents to traumatic stress questionnaire. Journal of Traumatic Stress.

Bean, T., Eurelings-Bontekoe, E. H. M., Derluyn, I., \& Spinhoven, P. (2004a), HSCL-37A user's manual Oegstgeest. (Available at http://www.centrum45.nl).
Bean, T., Eurelings-Bontekoe, E. H. M., Derluyn, I., \& Spinhoven, P. (2004b), SLE user's manual. Oegstgeest. (Available at http:// www.centrum45.nl).

Bean, T., Eurelings-Bontekoe, E. H. M., Derluyn, I., \& Spinhoven, P. (2004c). RATS user's manual. Oegstgeest. (Available at http:// www.centrum45.nl).

Bean, T., Mooijaart, A., Eurelings-Bontekoe, E. H. M. \& Spinhoven, P. (in press). Validation of the child behavior checklist for guardians of unaccompanied refugee minors. Child and Youth Services Review.

Bean, T., Mooijaart, A., Eurelings-Bontekoe, E. H. M. \& Spinhoven, P. (submitted). Validation of the teacher's report form for teachers of unaccompanied refugee minors.

Brugman, E., Reijneveld, S. A., Verhulst, F. C., \& Verloove-Vanhorick, S. P. (2001). Identification and management of psychosocial problems by preventive child health care. Archives of Pediatrics Adolescent Medicine, 155, 462-469.

Bukner, J. C., \& Baasuk, E. L. (1997). Mental disorders and service utilization among youths from homeless and Low-Income housed families. Journal of the American Academy of Child \& Adolescents Psychiatry, 36, 890-900.

Burns, B. J., Phillips, S. D., Wagner, H. R., Barth, R. P., Kolko, D. J., Campbell, Y., \& Landsverk, J. (2004). Mental health need and access to mental health services by youths involved with child welfare: A national survey. Journal of the American Academy of Child \& Adolescent Psychiatry, 43, 960-970.

Cauce, A. M., Domenech-Rodriguez, M., \& Paradise, M. (2002). Cultural and contextual influences in mental health help seeking: A focus on ethnic minority youth. Journal of Consulting \& Clinical Psychology, 70, 44-55.

Ciarrochi, J., Deane, F., Wilson, C., \& Rickwood, D. (2002). Adolescents who need help the most are the least likely to seek it: The relationship between low emotional competence and low intention to seek help. British Journal of Guidance and Counselling, 30, 173-188.

Cheng, A. T. (2001). Case definition and culture: Are people all the same? British Journal of Psychiatry, 179, 1-3.

Cohen, J. (1988). Statistical power analysis for the behavioral sciences (2nd ed). New York: Academic Press.

De Rosa, C. J., Montgomery, S. B., Kipke, M. D., Iverson, E., Ma, J. L., \& Unger, J. B. (1999). Service utilization among homeless and runaway youth in Los Angels, California: Rates and reasons. Journal of Adolescent Health, 24, 190-200.

Dutra, L., Campbell, L., \& Westen, D. (2004). Quantifying clinical judgment in the assessment of adolescent psychopathology: Reliability, validity and factor structure of the Child Behaviour Checklist for clinician report. Journal of Clinical Psychology, 60, 65-85.

Farmer, E. M., Burns, B. J., Phillips, S. D., Angold, A., \& Costello, E. J. (2003) Pathways into and through mental health services for children and adolescents. Psychiatry Services, 54, 60-66.

Ferdinand, R. F., Van der Ende, J., \& Verhulst, F. C. (2004). Parent-adolescent disagreement regarding psychopathology in adolescents from the general population as a risk factor for adverse outcome. Journal of Abnormal Psychology, 113, 198206.

Felsman, J., Leong, F., Johnson, M., \& Felsman, I. (1990). Estimates of psychological distress among Vietnamese refugees: Adolescents, unaccompanied minors, and young adults. Social Science \& Medicine, 31, 1251-1256.

Flaherty, J. A., Gaviria, F. M., Pathak, D., Mitchell, T., Wintrob, R., \& Richman, J. A. (1988). Developing instruments for crosscultural psychiatric research. Journal of Nervous and Mental Disease, 176, 257-263. 
Flisher, A. J., Kramer, R. A., Grosser, R. C., Alegria, M., Goodman, S. H., Greenwald, S., Horwitz, S. M., Moore, R. E., Narrow, W. E., \& Hoven, C. W. (1997). Correlates of unmet need for mental health services by children and adolescents. Psychological Medicine, 27, 1145-1154.

Ezpeleta, L., Keeler, G., Erkanli, A., Costello, J., \& Angold, A. (2001). Epidemiology of psychiatric disability in childhood and adolescence. Journal of Child Psychology \& Psychiatry \& Allied Disciplines, 42, 901-914.

Garland, A. F., Aarons, A. G., Brown, S. A., Wood, P. A., \& Hough, R. L. (2003). Diagnostic profiles associated with use of mental health and substance abuse services among high-risk youths. Psychiatric Services, 54, 562-564.

Garwood, M. M., \& Close, W. (2001). Identifying the psychological needs of foster children. Child Psychiatry \& Human Development, 32, 125-135.

Halvorsen, K. (2002). Separated children seeking asylum: The most vulnerable of all. Forced Migration Review, 12, 34-36.

Hodes, M. (2002). Implications for psychiatric services of chronic civilian strife: young refugees in the UK. Advances in Psychiatric Treatment, 8, 366-374.

Howard, M., \& Hodes, M. (2000). Psychopathology, adversity, and service utilisation of young refugees. Journal of the American Academy of Child \& Adolescents Psychiatry, 39, 368-377.

Hukkanen, R., Sournader, A., Bergroth, L., \& Piha, J. (1999). Psychosocial factors and adequacy of services for children in children's homes. European Child \& Adolescent Psychiatry, 8, 268-275.

Kataoka, S. H., Zhang, L., \& Wells, K. B. (2002). Unmet need for mental health care among U.S. children: variation by ethnicity and insurance status. American Journal of Psychiatry, 159, $1548-1555$

Kodjo, C., \& Auinger, P. (2004). Predictors for emotionally distressed adolescents to receive mental health care. Journal of Adolescent Health, 35, 368-373.

Kohli, R., \& Mather, R. (2003). Promoting psychosocial well-being in unaccompanied asylum seeking young people in the United Kingdom. Child \& Family Social Work, 8, 201-212.

Kinzie, J. D., Sack, W. H., Angell, R. H., Manson, S., \& Rath, B. (1986). The psychiatric effects of massive trauma on Cambodian children part one: The children. Journal of the American Academy of Child and Adolescent Psychiatry, 25, 370-376.

Kramer, T. L., Phillips, S. D., Hargis, M. B., Miller, T. L., Burns, B. J., \& Robbins, J. M. (2004). Disagreement between parent and adolescent reports of functional impairment. Journal of Child Psychology and Psychiatry and Allied Disciplines, 45, 248259.

Leaf, P. J., Alegria, M., Cohen, P., Goodman, S. H., Horwitz, S. M., Hoven, C. W., Vaden-Kiernan, N., Michael, W. E., \& Regier, D. A. (1996). Mental health service use in the community and schools: Results from the four-community MECA study. Journal of the American Academy of Child and Adolescent Psychiatry, 35, 889-897.

Lusitg, S., Kia-Keating, M., Knight, W. G., Geltman, P., Ellis, H., Kinizie, J. D., Keane, T., \& Saxe, G. N. (2004). Review of child and adolescent refugee mental health. Journal of the American Academy of Child \& Adolescents Psychiatry, 43, 24-36.

McMillen, J. C., Zima, B. T., Scott, L. D., Auslander, W. F., Muson, M. R., Ollie, M. T., \& Spitnagel, E. L. (2005). Prevalence of psychiatric disorders among older youths in the foster care system. Journal of the American Academy of Child \& Adolescent Psychiatry 44, 88-95.

Masser, D. (1992). Psychosocial functioning of Central American refugee children. Child Welfare, 71, 439-456.

Roberts, R. E., Attikison, C. C., \& Rosenblatt, A. (1998). Prevalence of psychopathology among children and adolescents. American Journal of Psychiatry, 155, 715-772.
Rousseau, C., Drapeau, A., Lacroix, L., Bagilishya, D., \& Heusch, N. (2005). Evaluation of a classroom program of creative expression workshops for refugee and immigrant children. Journal of Child Psychology and Psychiatry, 46, 180-185.

Sack, W., Him, C., \& Dickason, D. (1999). Twelve-year follow-up study of the Khmer youths who suffered massive war trauma as children. Journal of the American Academy of Child \& Adolescents Psychiatry, 38, 384-391.

Sack, W. H., Angell, R. H., Kinzie, J. D., \& Rath, B. (1986). The psychiatric effects of massive trauma on Cambodian children part two: The home and school. Journal of the American Academy of Child \& Adolescents Psychiatry, 25, 377-383.

Saunders, S. M., Resnick, M. D., Hoberman, H. M., \& Blum, R. W. (1994). Formal help-seeking behavior of adolescents identifying themselves as having mental health problems. Journal of the American Academy of Child and Adolescent Psychiatry, 33, $718-728$.

Sears, H. A. (2004). Adolescents in rural communities seeking help: who reports problems and who sees professionals? Journal of Child Psychology and Psychiatry, 45, 396-404.

Sheffield, J. K., Fiorenza, E., \& Sofronoff, K. (2004). Adolescents' willingness to seek psychological help: Promoting and preventing factors. Journal of Youth and Adolescence, 33, 495-507.

Silove, D., Steel, Z., McGorry, P., \& Drobny, J. (1999). Problems Tamil asylum seekers encounter in accessing health and welfare services in Australia. Social Science \& Medicine, 49, 951-956.

Sourander, A. (1998). Behavioural problems and traumatic events of unaccompanied refugee minors. Child Abuse and Neglect, 22, 719-727.

Sourander, A., Helstelä, L., Ristkari, T., Ikäheimo, K., Helenius, H., \& Piha, J. (2001). Child and adolescent mental health service use in Finland. Social Psychiatry \& Psychiatry Epidemiology, 36, 294-298.

Sourander, A., Santalahti, P., Haavisto, A., Piha, J., Ikaheimo, K., \& Helenius, H. (2004). Have there been changes in children's psychiatric symptoms and mental health service use? A 10-year comparison from Finland. Journal of the American Academy of Child \& Adolescents Psychiatry, 43, 1134-1144.

Smith, P., Perrin, S., Yule, W., Hacam, B., \& Stuvland, R. (2002). War exposure among children from Bosnia-Hercegovina: Psychologucal adjustment in a community Sample. Journal of Traumatic Stress, 15, 147-156.

Srebnik, D., Cauce, A. M., \& Baydar, N. (1996). Help-seeking pathways for children and adolescents. Journal of Emotional and Behavioral Disorders, 4, 210-221.

Steel, Z., Silove, D., Phan, T., \& Bauman, A. (2004). Long-term effect of psychological trauma on the mental health of Vietnamese refugees resettled in Australia: A population-based study. The Lancet, 360, 1056-1062.

Ten Have, M., Vollenbergh, W., Bijl, R., \& Ormel, J. (2002). Combined effect of mental disorder and low social support on care service use for menatl health problems in the Dutch general population. Psychological Medicine, 32, 311-323.

Tieman, W., van der Ende, J., \& Verhulst, F. C. (2005). Psychiatric disorders in young adult Intercountry Adoptees: An epidemiological study. American Journal of Psychiatry, 162, 592-598.

US Department of Health and Human Services (1999). Mental health; A report of the surgeon general. Chapter 3 . Children and mental health. Surgeon general,U.S. Public Health Service. Retrieved December 12, 2004 from http://www.surgeongeneral.gov/library/mentalhealth/home.html

Verhulst, F. C., van der Ende, J., \& Koot, H. M. (1996). Manual for the child behavior checklist (in Dutch). Sophia, Rotterdam: Department of Child and Adolescent Psychiatry, Erasmus Medical Centre. 
Verhulst, F. C., van der Ende, J., \& Koot, H. M. (1997). Manual for the teacher's report form (in Dutch). Sophia, Rotterdam: Department of Child and Adolescent Psychiatry, Erasmus Medical Centre.

Verhulst, F. C., \& van der Ende, J. (1997). Factors associated with child mental health service use in the community. Journal of American Academy of Child and Adolescent Psychiatry, 36, 901-909.

Weisz, J. R., \& Hawley, K. M. (2002). Developmental factors in the treatment of adolescents. Journal of Consulting and Clinical Psychology, 70, 21-43.

Wu, P., Hoven, C. H., Bird, H. R., Moore, R. E., Cohen, P., Alegria, M., Dulcan, M. K., Goodman, S. H., McCue-Horwitz, S., Lichtman, J. H., Narrow, W. E., Rae, D. S., Regier, D. A., \& Roper, M. T. (1999). Depressive and disruptive disorders and mental health service utilization in children and adolescents. Journal of the American Academy of Child \& Adolescents Psychiatry, 38, 1081-1092.
Yeh, M., \& Weisz, J. R. (2001). Why are we here at the clinic? Parent-child (dis)agreement on referral problems at outpatient treatment entry. Journal of Consulting and Clinical Psychology, 69, 1018-1025.

Yeh, M., Hough, R. L., McCabe, K., Lau, A., \& Garland, A. (2004). Parental beliefs about the causes of child problems: Exploring racial/ethnic patterns. Journal of the American Academy of Child and Adolescent Psychiatry, 43, 605-612.

Zwaanswijk, M., Verhaak, P. F., Bensing, J. M., van der Ende, J., \& Verhulst, F. C. (2003a). Help-seeking for emotional and behavioural problems in children and adolescents: a review of recent literature. European Child Adolescent Psychiatry, 12, 153-161.

Zwaanswijk, M., Verhaak, P. F., Bensing, J. M., van der Ende, J., \& Verhulst, F. C. (2003b) Factors associated with adolescent mental health service need and utilization. Journal of the American Academy of Child and Adolescent Psychiatry, 42, 692-700. 